International Journal of Pure and Applied Mathematics

Volume 87 No. $6 \quad 2013,835-844$

ISSN: 1311-8080 (printed version); ISSN: 1314-3395 (on-line version)

url: http://www.ijpam.eu

doi: http://dx.doi.org/10.12732/ijpam.v87i6.12

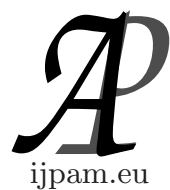

\title{
A STUDY ON THE COVERING NUMBER OF GENERALIZED JAHANGIR GRAPHS $\boldsymbol{J}_{s, m}$
}

\author{
D. Angel ${ }^{1}$, A. Amutha ${ }^{2}$ \\ ${ }^{1,2}$ Department of Mathematics \\ Sathyabama University \\ Chennai, 119, INDIA
}

\begin{abstract}
A set $S$ of vertices of a graph $G=(V, E)$ is called a vertex cover, if each edge in $\mathrm{E}$ has at least one end point in $S$ and the minimum cardinality taken over all vertex covering sets of $G$ is called the covering number of $G$ denoted by $\beta(G)$. In this paper, we study some results on vertex covering, edge covering, strong and weak covering, and inverse covering numbers denoted by $\beta(G), \beta^{\prime}(G), s \beta(G), w \beta(G), \beta^{-1}(G)$ respectively for generalized Jahangir graphs $J_{s, m}$. We have also characterized the graphs which are invertible.
\end{abstract}

AMS Subject Classification: $05 \mathrm{C} 70$

Key Words: vertex cover, edge cover, inverse cover, strong cover and generalized Jahangir graphs

\section{Introduction}

The vertex cover problem has many real world applications. This is one of the best studied graph problems in theoretical computer science which is a special class of the set cover problem. An interesting field of application of the vertex cover problem is in network design and also in bio-informatics, in the

Received: September 6, 2013

(c) 2013 Academic Publications, Ltd. url: www.acadpubl.eu 
construction of polygenetic trees, in phenotype identification and in analysis of micro array data. It is also useful in routing algorithms and in fault-tolerance algorithms. It is well known that the vertex cover problem is $N P$ complete for general graphs. Determining how well we can approximate vertex cover is one of the outstanding open problems in the complexity of approximation. While vertex cover has a trivial 2-approximation algorithm, no better algorithms are known. The best approximation algorithm currently known for arbitrary graph is due to Karakostas. In cubic graphs, the minimum vertex cover problem is known to be APX approximable complete and NP hard in planar graphs, and admits a polynomial time approximation scheme in planar graphs [1]. On the other hand, minimum vertex cover is polynomial for several classes of graphs such as bipartite, chordal and graphs with bounded tree width etc. Therefore many attempts have been made to find the lower and upper bounds and exact values of $\beta(G)$ for special classes of graphs. Recently, Babak et. al. characterized minimum vertex cover in generalized Petersen graphs [4] and Madhavi. L et. al. obtained exact values of minimum vertex cover and minimum edge cover in Mangoldt graphs [9]. Various graph characterizations have been done on covering relating to domination parameters and matching numbers and new graph parameters such as inverse vertex cover are being defined [5]. Motivated by the various applications of vertex covering we are interested in finding the covering parameters in generalized Jahangir graphs $J_{s, m}$.

\section{Preliminaries}

A graph $G=(V, E)$ is a set of points $V$ called vertices and a collection $E$ of unordered pairs of vertices called edges. A graph $G$ is said to be a connected graph, if every pair of vertices of $G$ has a path from one vertex to another. All the graphs considered here are finite, connected and undirected with no loops and multiple edges. A matching in a graph is a set of pair wise non adjacent edges and a maximum matching is a matching that contains the largest possible number of edges. A perfect matching is a maximum matching which matches all vertices of the graph. It is also called a one factor. If for every vertex, there is a near perfect matching that omits only one vertex, then that graph is called factor critical. A graph $G$ is bipartite if and only if it does not contain any odd cycles. 


\section{Overview of the Paper}

We have already studied the covering parameters on three fence graphs such as Mobius ladder graphs, circular ladder graphs and ladder graphs [2] and also given a characterization of trees using clique cover and the complement of a tree [3]. In this paper, we extend our study on covering numbers to generalized Jahangir graphs $J_{s, m}$.

\subsection{Minimum Vertex Cover Problem}

A set $S$ of vertices of a graph $G=(V, E)$ is called a vertex cover, if each edge in $E$ has at least one end point in $S$. The minimum vertex cover is a vertex cover of smallest size. The cardinality of the minimum vertex cover set is called the covering number of $G$, denoted by $\beta(G)$. Finding the minimum vertex cover is called the vertex cover problem.

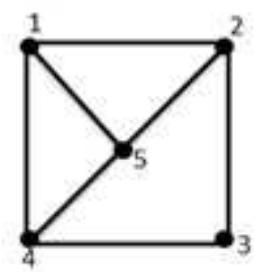

Figure 1: Minimum vertex cover set $=\{1,3,5\}$

\subsection{Minimum Edge Cover Problem}

A set $C$ of edges of a graph $G$, such that each vertex of $G$ is incident with at least one edge in $C$ is called an edge cover. The minimum edge cover is an edge cover of smallest size and the cardinality of the minimum edge cover set is called the edge covering number denoted by $\beta^{\prime}(G)$. Finding the minimum edge cover is called the edge covering problem.

\subsection{Minimum Inverse Vertex Cover Problem}

Let $D$ be a minimum vertex covering of $G$. A set $S \subseteq V-D$ which is a vertex covering of $G$ is called an inverse vertex covering of $G$ with respect to the covering $D$. Then the inverse vertex covering number $\beta^{-1}(G)$ is the order of smallest inverse vertex covering of $G$. Note that $\beta^{-1}(G)$ need not exist for 


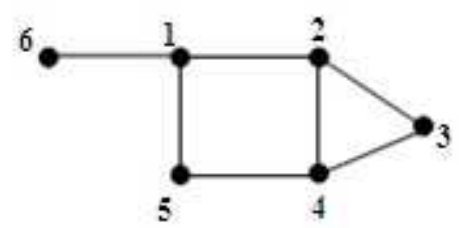

Figure 2: Minimum edge cover set $=\{(1,6),(2,3),(4,5)\}$

every graph. For example, the cycle graph on odd number of vertices has no inverse vertex cover. A graph $G$ is said to be invertible if $G$ admits an inverse vertex covering [5]. For figure 3, the minimum vertex cover set $D=\{1,3,5,7\}$ or $\{2,4,6,8\}$ and $\beta(G)=4$, whereas, the minimum inverse vertex cover set $V-D=\{2,4,6,8\}$ or $\{1,3,5,7\}$ with respect to $D$ and therefore, $\beta^{-1}(G)=4$.

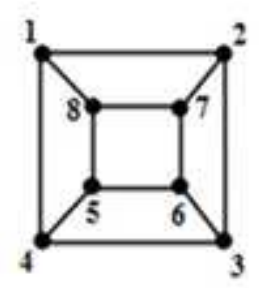

Figure 3: Minimum inverse vertex cover set $=\{24,6,8\}$ or $\{1,3,5$, 7\}

\subsection{Jahangir Graphs $J_{s, m}$}

Jahangir graphs $J_{s, m}$ for $s \geq 2, m \geq 2$, is a graph on $s m+1$ vertices consisting of a cycle $C_{s m}$ with one additional vertex which is adjacent to $m$ vertices of $C_{s m}$ at distance $s$ to each other on $C_{s m}$. Figure 4 shows Jahangir graph $J_{2,8}$. This graph $J_{2,8}$, appears on the Jahangir's tomb in his mausoleum. It lies in 5 kilometer north-west of Lahore, Pakistan across the River Ravi. His tomb was built around 1637 A.D. by his Queen Noor Jehan and his son Shah-Jehan, the emperor who constructed one of the wonders of the world Taj Mahal in India. It has a majestic structure made of red sand-stone and marble. The definition of Jahangir graph $J_{2, m}$ was introduced by Surahmat and Tomescu in 2006 [7]. 


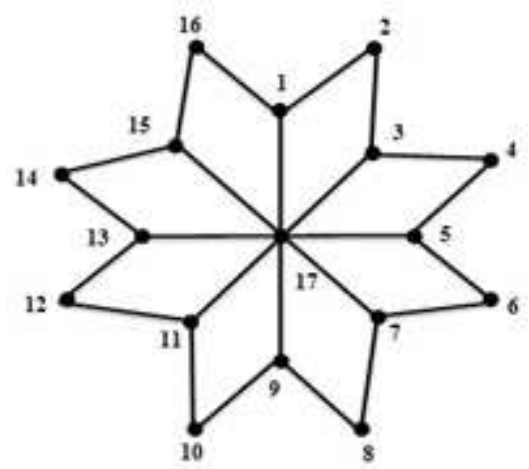

Figure 4: $J_{2,8}$

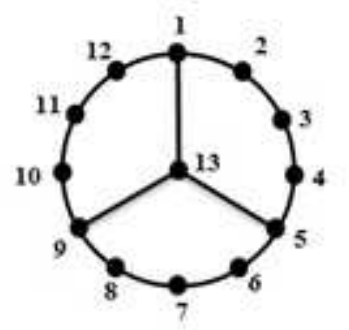

Figure 5: $J_{4,3}$

\subsection{Remark}

Let $s m+1$ be the label of the center vertex and $1,2,3, \ldots, s m$ be the label of the vertices that are incident clockwise on cycle $C_{s m}$ so that degree of the vertex labelled 1 is three.

\section{Main Results}

Theorem 4.1. Let $G$ be a Jahangir graph $J_{s, m}$. Then $\beta(G)=\left\lfloor\frac{|V|}{2}\right\rfloor$, if $s$ is even and $m \geq 2$. 
Proof. Let $G$ be a Jahangir graph $J_{s, m}$. Let $D$ be the minimum vertex cover set of $G$. To prove: $\beta(G)=\left\lfloor\frac{|V|}{2}\right\rfloor$, if $s$ is even.

Since $s$ is even, sm is even and hence $C_{s m}$ is an even cycle. Choosing alternate vertices on the even cycle $C_{s m}$ starting from the vertex labelled 1 , we see that $D$ covers all edges on $C_{s m}$ as well as the edges incident on the middle vertex. This implies, $\beta\left(J_{s, m}\right)=\beta\left(C_{s m}\right)=\frac{s m}{2}$.

Therefore, $\beta\left(J_{s, m}\right)=\frac{s m}{2}=\frac{s m}{2}+\frac{1}{2}-\frac{1}{2}=\frac{|V|}{2}-\frac{1}{2}=\left\lfloor\frac{|V|}{2}\right\rfloor$.

Theorem 4.2. Let $G$ be a Jahangir graph $J_{s, m}$. Then $\beta(G)=\left\lceil\frac{|V|}{2}\right\rceil$, if $s$ is odd and $m$ is even.

Proof. Let $G$ be a Jahangir graph $J_{s, m}$. Let $D$ be the minimum vertex cover set of $G$.

To prove: $\beta(G)=\left\lceil\frac{|V|}{2}\right\rceil$, if $s$ is odd and $m$ is even.

Since $s$ is odd and $m$ is even, $s m$ is even.

For covering the edges on $C_{s m}$ we choose alternate vertices on the even cycle $C_{s m}$.

But since $s m$ is even, $\beta\left(C_{s m}\right)=\frac{s m}{2}$.

Now since all degree three vertices are not in $D$, we choose the middle vertex labeled $s m+1$ also, such that all the edges of $G$ are covered. Therefore $\beta\left(J_{s, m}\right)=$ $\beta\left(C_{s m}\right)+1$.

This implies $\beta\left(J_{s, m}\right)=\frac{s m}{2}+1=\frac{s m}{2}+\frac{1}{2}+\frac{1}{2}=\frac{|V|}{2}+\frac{1}{2}=\left\lceil\frac{|V|}{2}\right\rceil$.

Theorem 4.3. Let $G$ be a Jahangir graph $J_{s, m}$. Then $\beta(G)=\frac{|V|}{2}+1$, if both $s$ and $m$ are odd.

Proof. Let $G$ be a Jahangir graph $J_{s, m}$. Let $D$ be the minimum vertex cover set of $G$.

To prove: $\beta(G)=\frac{|V|}{2}+1$, if both $s$ and $m$ are odd.

Since $s$ and $m$ are is odd, $s m$ is also odd. For covering the edges on $C_{s m}$ we choose the altenate vertices on $C_{s m}$ and as $C_{s m}$ is an odd cycle, we have $\beta\left(C_{s m}\right)=\frac{s m+1}{2}$.

Now since all degree three vertices are not in $D$, we choose the middle vertex labeled $s m+1$ also, such that all the edges of $G$ are covered. Hence $\beta\left(J_{s, m}\right)=$ $\beta\left(C_{s m}\right)+1$.

This implies $\beta\left(J_{s, m}\right)=\frac{s m+1}{2}+1=\frac{|V|}{2}+1$.

Theorem 4.4. Let $G$ be a Jahangir graph $J_{s, m}$. Then $\beta(G)=\beta^{\prime}(G)$, if $s$ is odd and $m$ is even. 
Proof. Let $G$ be a Jahangir graph $J_{s, m}$. To prove: $\beta(G)=\beta^{\prime}(G)$, if $s$ is even and $m$ is odd. Since $s$ is even, $s m$ is even. So $C_{s m}$ will contain even number of edges and $\beta^{\prime}\left(C_{s m}\right)=\frac{s m}{2}$. We choose the alternate edges on $C_{s m}$ so that all the vertices on $C_{s m}$ are covered and for covering the middle vertex we choose one more edge. Therefore $\beta^{\prime}\left(J_{s, m}\right)=\beta^{\prime}\left(C_{s m}\right)+1$. Hence $\beta^{\prime}\left(J_{s, m}\right)=\frac{s m}{2}+1$.

This implies from theorem 4.2, $\beta^{\prime}\left(J_{s, m}\right)=\left\lceil\frac{|V|}{2}\right\rceil=\beta(G)$. Hence the proof.

Theorem 4.5. Let $G$ be a Jahangir graph $J_{s, m}$. Then $\beta(G)=\beta^{\prime}(G)-1$, if $s$ is even and $m \geq 2$.

Proof. Let $G$ be a Jahangir graph $J_{s, m}$. To prove: $\beta(G)=\beta^{\prime}(G)-1$, if $s$ is even and $m \geq 2$. Since $s$ is even, $s m$ is even. So $C_{s m}$ will contain even number of edges and $\beta^{\prime}\left(C_{s m}\right)=\frac{s m}{2}$. We choose the alternate edges on $C_{s m}$ so that all the vertices on $C_{s m}$ are covered and for covering the middle vertex we choose one more edge. Therefore $\beta^{\prime}\left(J_{s, m}\right)=\beta^{\prime}\left(C_{s m}\right)+1$. Hence $\beta^{\prime}\left(J_{s, m}\right)=\frac{s m}{2}+1$. From theorem 4.1, $\beta^{\prime}\left(J_{s, m}\right)=\beta\left(J_{s, m}\right)+1$.

This implies $\beta\left(J_{s, m}\right)=\beta^{\prime}\left(J_{s, m}\right)-1$. Hence the result.

Theorem 4.6. Let $G$ be a Jahangir graph $J_{s, m}$. Then $\beta(G)=\beta^{\prime}(G)+1$, if both $s$ and $m$ are odd.

Proof. Let $G$ be a Jahangir graph $J_{s, m}$. To prove: $\beta(G)=\beta^{\prime}(G)+1$, if both $s$ and $m$ are odd. Since both $s$ and $m$ are odd, $J_{s, m}$ contains an odd cycle $C_{s m}$. We choose all the alternate edges on $C_{s m}$ and another edge which covers both the center vertex and the left out vertex in $C_{s m}$. Therefore, $\beta^{\prime}\left(J_{s, m}\right)=\frac{s m+1}{2}=\frac{|V|}{2}$.

But $\beta\left(J_{s, m}\right)=\frac{|V|}{2}+1$. This implies, $\beta\left(J_{s, m}\right)=\beta^{\prime}\left(J_{s, m}\right)+1$. Hence the proof.

Theorem 4.7. Let $G$ be any graph. Then $G$ is invertible if and only if there exists no edge $x=u v$ in $G$ such that both $u$ and $v$ are in the minimum vertex cover set of $G$.

Proof. Let $G$ be a graph. Assume $G$ is invertible.

Let $D$ be the minimum vertex cover set of $G$.

To prove: There exists no edge $x=u v$ in $G$ such that both $u$ and $v$ are in $D$. Suppose if there exists an edge $x=u v$ in $G$ such that both $u$ and $v$ are in $D$. Then clearly $V-D$ will not contain $u$ and $v$. This implies that $x=u v$ is not covered by $V-D$. That is, there is no inverse vertex cover for $G$ and so $G$ is not invertible, which is a contradiction to our assumption. Therefore there 
exists no edge $x=u v$ in $G$ such that both $u$ and $v$ are in $D$.

Conversely, let us assume that there exists no edge $x=u v$ in $G$ such that both $u$ and $v$ are in $D$. To prove: $G$ is invertible.

By our assumption, for any $u, v \in V$, if $u \in D$ then $v \in V-D$ and vice versa. This implies that $V-D$ is a vertex cover of $G$. Hence $G$ is invertible.

Theorem 4.8. Let $G$ be any graph. Then $G$ is not invertible if $G$ contains odd cycles.

Proof. Let $G$ be any graph and $D$ be the minimum vertex cover set of $G$. If there is an odd cycle in $G$ then clearly there is at least one edge $x=u v$ in $G$ such that both $u$ and $v$ are in $D$. Hence from theorem 4.6, $G$ is not invertible.

Theorem 4.9. Let $G$ be a Jahangir graph $J_{s, m}$. Then, for all $m \geq 2$, $\beta^{-1}(G)=\beta^{\prime}(G)$, if $s$ is even.

Proof. Let $G$ be a Jahangir graph $J_{s, m}$. To prove: $\beta^{-1}(G)=\beta^{\prime}(G)$ if $s$ is even and for $m \geq 2$.

Since $s$ is even, $C_{s m}$ is an even cycle.

Hence there exists no edge $x=u v$ in $G$ such that both $u$ and $v$ are in the minimum vertex cover set of $G$.

This implies from theorem 4.7 that $J_{s, m}$ is invertible.

So inverse vertex cover exists.

Therefore, we have, $\beta^{-1}(G)=|V|-\beta(G)=s m+1-\frac{s m}{2}=\frac{s m}{2}+1=\beta^{\prime}(G)$. Hence the proof.

\section{Observations 4.10.}

(i) If $s$ is even then $J_{s, m}$ does not contain any odd cycle and hence is bipartite.

(ii) For all $s \geq 2$ and $m \geq 2$, each $J_{s, m}$ contains $C_{s+2}$ as an induced subgraph.

\section{Strong, Weak and Balanced Vertex Covers}

A vertex $v$ in a graph $G=(V, E)$ is said to be strong if $\operatorname{deg}(v) \geq \operatorname{deg}(u)$ for every $u$ adjacent to $v$ in $G$. A set $S \subseteq V$ is said to be strong if every vertex in $S$ is a strong vertex in $G$. For an edge $x=u v, v$ strongly covers the edge $x$ if $\operatorname{deg}(v) \geq \operatorname{deg}(u)$ in $G$. A set $S \subseteq V$ is a strong vertex cover $[S V C]$ if every edge in $G$ is strongly covered by some vertex in $S$. The strong vertex covering 
number is the minimum cardinality of an $S V C$ denoted by $s \beta(G)$. For an edge $x=u v, v$ weakly covers the edge $x$ if $\operatorname{deg}(v) \leq \operatorname{deg}(u)$ in $G$. A set $S \subseteq V$ is a weak vertex cover $[W V C]$ if every edge in $G$ is weakly covered by some vertex in $S$. The weak vertex covering number is the minimum cardinality of a $W V C$ denoted by $w \beta(G)$. One can easily note that a vertex $v \in V$ is balanced if, and only if, $\operatorname{deg}(v)<\operatorname{deg}(u)$ and $\operatorname{deg}(v)>\operatorname{deg}(w)$ for some $u, w$ adjacent to $v$. If a balanced vertex set $S \subseteq V$ covers all the edges of $G$ then $S$ is called a balanced vertex cover $[B V C]$ denoted by $B \beta(G)[8]$.

In figure 6 , The only strong vertex in $J_{2,4}$ is the vertex labelled 9 and this vertex alone does not cover all the edges of $J_{2,4}$. Hence no strong vertex cover exists for $J_{2,4}$. The weak vertex set for $J_{2,4}=\{2,4,6,8\}$ and these vertices do not cover all the edges of $J_{2,4}$. Hence $s \beta(G)=w \beta(G)=0$. The balanced vertices of $J_{2,4}$ are $=\{1,3,5,7\}$ and all the vertices in this set cover all edges of $J_{2,4}$ and hence form a balanced vertex cover for $J_{2,4}$.

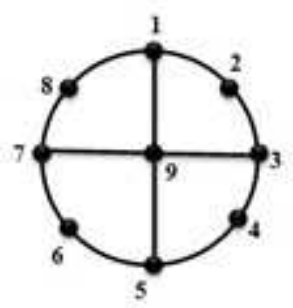

Figure 6: $J_{2,4}$

Proposition 5.1. Let $G$ be a Jahangir graph $J_{s, m}$. Then $s \beta(G)=$ $w \beta(G)=0$ for all $s \geq 2$ and $m \geq 2$.

Proposition 5.2. Let $G$ be a Jahangir graph $J_{s, m}$. Then for all $m \geq 2$, (i) $B \beta(G)=0$, if $s>2$ (ii) $B \beta(G)=\beta(G)$, if $s=2$.

\section{Conclusion}

In the previous sections, we have calculated the exact values of the parameters $\beta(G), \beta^{\prime}(G), \beta^{-1}(G), s \beta(G), w \beta(G)$ and $B \beta(G)$ for generalized Jahangir graphs $J_{s, m}$ and hence obtained the relations between them. Also we have given a characterization of invertible graphs. We can also extend our study on covering numbers to other cycle generated graphs such as theta graphs and generalised friendship graphs. 


\section{References}

[1] Alimonti. P and Kann., V, Some APX-completeness results for cubic graphs, Theoretical Computer Science, 2000, Volume 237, Pages 123-134.

[2] Amutha. A, Angel. D, On the Covering Number of Fence Graphs, Proceedings of the National Seminar on Discrete Mathematics and its Applications, M.E.S. College, Nedumkandam, February 06-08, 2012, Pages 61-64.

[3] Amutha. A, Angel. D, Characterization of Trees using Covers, Proceedings of the National Conference on Mathematical and Computational Modelling, Sathyabama University, June 27-29, 2012, Pages 148-152.

[4] Babak, Pooya and Ebadollah, On minimum vertex cover of Generalized Petersen graphs, Australasian Journal of Combinatorics, 2007, Volume 40, Pages 253-264.

[5] Bhat. P.G, and R.S. Bhat, Inverse Independence Number of a Graph, International Journal of Computer Applications, 2012, Volume 42, Pages 9-13.

[6] Geir Agnarsson and Raymond Green Law Graph Theory: Modeling, Applications, and Algorithms, Pearson/Prentice Hall, 2007, Pages 334-351.

[7] Kashif Ali, Edy Tri Baskoro, I. Tomescu, On the Ramsey numbers for paths and generalized Jahangirs, $J_{s, m}$, Bull. Math. Soc. Sci. Math. Roumanie Tome, 2008, Volume 51, pages 177-182.

[8] Kamath.S, R. S. Bhat, On strong (weak) independent sets and vertex coverings of a graph, Discrete Mathematics, 2007, Volume 307, Pages 11361145.

[9] Madhavi. L and Maheswari. B, Edge Cover Domination in Mangoldt Graph, Momona Ethiopian Journal of Science, 2011, Volume 3, Pages 3751. 